Espinoza d. $y$ cols.

Rev. Chll. Pedlatr. 64 (6); 364-370, 1993

\title{
Colonización bacteriana anormal del intestino delgado alto en escolares de nivel socioeconómico alto y bajo
}

\author{
Julio Espinoza M.1; Oscar Brunser T.1; Magdalena Araya Q. ${ }^{2}$
}

\begin{abstract}
Abnormal microbiological contamination of the small intestine among school-age children of the high and low socioeconomic strata
\end{abstract}

\begin{abstract}
Microbiological conlamination of the enviroment induces chronic enviromental enteropalhy, characterized by mild to moderate morphological and funclional changes of the small inteslinal mucosa. Bacteriol colonizalion wos evaluated in 31 ] school aged children 1155 of the high [H-SES) and 150 of the low [L-SES! socioeconomic strolo! by means of the breath $\mathrm{H}_{2}$ test. Somples were obtained after overnight fast and every 15 minules for 240 minutes following the ingeslion of $150 \mathrm{mg} \cdot \mathrm{kg}$ lactulose. A health questiongire that included information about lood and medicines ingeslion, alcohol consumption, smoking and intolerance to loodstults wos also applied. In $30 \%$ of the children bosal $\mathrm{H}_{2}$ excretion was above $10 \mathrm{ppm}$. In $18,1 \%$ of the H.5ES and $4.5 \%$ of the $1.5 E S$ (p $<0.0001$ ) $\mathrm{H}_{2}$ excietion was higher than $20 \mathrm{ppm}$ in the first 90 minutes. Colonic peaks $>20$ pam between 90 and 240 minutesl were detecied in $78.7 \%$ of the H.SES and $40,4 \%$ of the L.SES ip < 0.0001 ). In 18.7\% and 48.7\% of the H-SES and I-SES, respeclively, curves were flat ibasal excielion $<$ $10 \mathrm{ppm}$ and increments < $20 \mathrm{ppm}$ ). Chronic environmental enteropathy is alieady piesent in school age children. The lower breath $\mathrm{H}_{2}$ excretion observed in low socioeconomic individuals may be due to dilerences in quality and quantity of the diel.
\end{abstract}

[Key words: enleropathy, microbiological contomination, breath $\mathrm{H}_{2}$ excretion, lactulose, schoologe children.]

Una de las características de los países subdesarrollados es el deficiente saneamiento microbiológico del ambiente, que se traduce en altas incidencias de diarrea y de ocras infecciones'. En el tubo digestivo la contaminación microbiológica produce alteraciones morfológicas y funcionales que fueron descritas en habitantes de áreas tropicales, por lo que se denominaron enteropatia tropical2, 3. Estas alteraciones también ocurren en zonas templadas y el grado de contaminación modifica su intensidad. Así, estas lesiones eran máximas en paises como India o Haitf y eran mucho menores en Rhodesia (actualmente Zimbabwe) y en Chile ${ }^{4-7}$. Debido a que la relación con la contaminación del ambiente es el factor más importante se la denomina actual-

1. Unidad de Gastroenterología, Instiruto de Nutrición y Tecnología de los Alimentos (INTA), L'niversidad de Chile.

Financiado por el Proyecto Fondecyt 91-1043. mente enteropalia ambiental crónica ${ }^{8}$. Estudios realizados en Santiago en adultos jóvenes de nivel socioeconómico bajo (NSE-B) demostraron alteraciones histológicas y de la absorción de nutrientes de intensidad leve a moderada. El cultivo del jugo intestinal reveló contaminación del lumen yeyunal alto por anaerobios y microaerófilos. La ingesta de cantidades habituales de fibra dietaria se ascció a pérdidas desproporcionadas de nutrientes por las deposiciones. La demostración de estos hallazgos requirió de métodos invasores (estudios de balance, de perfusión con sonda de triple lumen y biopsias de la mucosa yeyunal). La enteropalía ambiental crónica puede repercutir negativamente sobre el estado nutricional10.

Otros estudios demostraron que preescolares de rivel socioeconómico alto (NSE-A) sufrían menos episodios de diarrea que los de NSE-B, pero tenf́an mayores niveles de portación de algunos enteropatógenos bacterianos ${ }^{11}$. También se demastró que la piel de las manos de las personas 
que manipulaban alimentos en el hogar y algunos alimentos de consumo habitual estaban altamente contaminados con bacterias fecales, independientemente det nivel socioeconomico ${ }^{12}$. El tránsito de enteropatógenos por el tracto digestivo se pesquisó desde los primeros días de vida y no se correlacionó con el nivel socioeconómico, lo que sugiere que depende principalmente del medio ambiente que rodea al sujeto al nacer ${ }^{13}$.

En la infancia no es posible usar métodos invasores, razón por la que la enteropatía ambiental crónica no ha sido cstudiada en esta edad. La medición de la excreción de $\mathrm{H}_{2}$ en cl aire espirado permitc explorar algunos de estos aspectos, ya que las variaciones de su concentración después de ingerir una sustancia no absorbible (como la lactulosa) reflejan la metabolización del sustrato por la flora intestinal residente o por cualquier colonización anormal ${ }^{14-17}$. El objetivo de estc estudio fue evaluar la presencia de contaminación microbiológica del intestino alto en precscolares y escolares.

\section{Sujetos y procedimientos}

Se seleccionaron escuelas de los niveles socioeconómicos alto y bajo. Después de recibir la autorización de los establecimientos educacionales, se abluvo el consentimiento escrito de los padres y la aceptación de participar de los alumnos. El nivel socioeconómíco se detcrminó mediante la escala de Graffar adaptada para población urbana de Chile ${ }^{10} .19$.

Se fommaron dos grupos de ambos sexos distribuidos entrc los 4 y los 14 años de edad: 155 de NSE-A y 156 del XSE-B (tabla 1). Las madres fueron instruidas para dar al niño aliuncntación liviana el día antes del examen, incluyen. do una comida pobre en fibra la noche anterior. Los escolares Jebían concurit en ayunas y los de menor edad fueron acompañados por su madre.

Se compleló un cuestionario precodificado acerca de la salud del niño, intolerancia a alimentos, y consumo de alcohol, tabaco o medicamentos. No ingresaron al estudio sujetos que hubieran recibido antibiótjous en los 15 días previos. La ingestión de alimentos se cualué mediante un recordatorio de 24 horas. El examen fisico incluía medicjones de peso y talla; los resultados fueron comparados con el estándar OMS NCHS $^{20}$ y expresados como puntuación Z21.

Para la prueba de excteción de $H_{2}$ se obtuvo una muestra basal, previa a la administración de $150 \mathrm{mg} \cdot \mathrm{kg}$ de peso de laciulosa analítica (Sigma Chemical Co., St. Louis, Mo. LSA.). A continuación se obtuvieron muestras cada 15 minutos por 6 horas, empleando bolsas de Mylar con válvula de entrada y saljidaló, 17 . El $\mathrm{H}_{2}$ se midió el mismo día en un cromalógrafo Quiniron Micro Lyzer Modeio 12.

Los resullados se analizaron con el paquete estadístico SAS (SAS Institute, Cary, NC. USA), usando promedios, desviaciones estándar, chi cuadrado y prueba $\mathrm{t}$ de Sudent ${ }^{2 t}$.

\section{Resultados}

Todos los sujetos estaban dentro de los márgenes normales o mostraban desviaciones moderadas en su peso corporal. En el NSE-B algunos niños tenían bajo peso para la edad; el examen físico demostró alta prevalencia de piezas dentales con caries o faltantes y mayor número de infecciones cutáneas, en tanto que los de NSE-A tendían ser más corpulentos y, algunos, obesos.

La encuesta de ingestión de 24 h reveló que para los alumnos de NSE-B el desayuno y el almuerzo que entrega la escuela representaron una proporción importante de la alimentación diaria; $47.4 \%$ no ingería otros alimentos (particularmente en la noche). Esta cifra era mucho menor $(6,5 \%)$ en el NSE-A. Las cantidades de fjbra dietaria eran semejantes en ambos niveles sociocconónicos; los niños de NSE-A alto ingerían más proteínas y energía.

En las mediciones basales, la excreción de más de $10 \mathrm{ppm}$ de $\mathrm{H}_{2}$ en el aure espirado fueron significativamente más frecuentes en el NSE-A ( $p<0,01$ ) (tabla 2). Si se usa como índice de fermentación yeyunal el aumento de más de $20 \mathrm{ppm}$ de $\mathrm{H}_{2}$ por sobre el valor basal en los primeros 90 minutos despues de ingerir la lactulosa, éste también fue más frecuente en los escolares de NSE-A $(28 / 155=18,1 \%$ vs $7 / 156=$ $4,5 \% ; p<0,0001\}$.

Para definir la cúspide o máximo colónico se considera la excreción de más de $10 \mathrm{ppm}$ de $\mathrm{H}_{2}$ sobre el nivel basal a los 90 a 240 minutos después de ingerir la lactulosa. En el NSE-A y en el NSE-B hubo cúspide colónica en 124/155 $(81,0 \%)$ y $76 / 156(49,3 \%)$, respectivamente

\section{Tabla 1}

Distribución de la edad de los escolares de nivel socioeconómico alto (NSE-A) y bajo (NSE-B) a los que se midio el hidrógeno espirado

\begin{tabular}{ccc}
\hline Edad (años) & NSE-A & NSE-B \\
\hline $4 \cdot 6$ & 9 & 14 \\
$6 \cdot 8$ & 32 & 34 \\
$8 \cdot 10$ & 30 & 32 \\
$10 \cdot 12$ & 36 & 32 \\
$12 \cdot 14$ & 37 & 32 \\
$>14$ & 11 & 12 \\
\hline Total & 155 & 156 \\
\hline
\end{tabular}


Tabla 2

Excreción basal > $10 \mathrm{ppm}$ de hidrógeno espirado antes de ingerir lactulosa en escolares de niveles socioeconómicos alto y bajo

\begin{tabular}{llc} 
& NSE alto $(n: 155)$ & NSE baja $(\mathbf{n}: 156)$ \\
\hline Hombres & $44^{*}$ & 21 \\
Mujeres & $18^{*}$ & 21 \\
Total & $62(40,0 \%)^{* *}$ & $42(26,9 \%)^{* *}$ \\
\hline$* \chi^{2}=20,97 ;$ & $p<0,00000$ & \\
$* * \chi^{2}=5,97 ;$ & $P<0,01452$
\end{tabular}

NSE: nivel sacioeconómico

( $p<0,00000$ ). Esta cúspide también se puede expresar como la elevación del $\mathrm{H}_{2}$ sobre 20 ppm entre los 90 y los 240 minutos de observación. En este caso también había diferencias significativas entre ambos NSE (tabla 3). En una proporción de los sujetos no se observo cúspide colónica (tabla 3). Esto no presupone necesariamente ausencia de cúspide yeyunal, ya que $6(10,2 \%)$ escolares de NSE-B que no la presentaban tuvieron excreciones de $\mathrm{H}_{2}$ superiones a $20 \mathrm{ppm}$ durante los primeros 90 minutos (tabla 3).

Algunas curvas de excreción de $\mathrm{H}_{2}$ en el aire espirado fueron planas, con niveles basales inferiores a $10 \mathrm{ppm}$ y sin elevaciones superiores a $20 \mathrm{ppm}$ durante todo el período de observación (tabla 4). En esta tabla aparece también la proporción de curvas con cúspide colónica superior a $20 \mathrm{ppm}$ entre $\operatorname{los} 90$ y los $240 \mathrm{minu}-$ tos, así como aquellas que tenían niveles iniciales de excreción de $\mathrm{H}_{2}$ superiores $\mathrm{o}$ inferiores a $10 \mathrm{ppm}$.

En la tabla 5 se analiza la relación entre la ingesta de alimentos, incluyendo fibra, y los valores de excreción de $\mathrm{H}_{2}$; se observaron diferencias entre los grupos para las diferentes formas de expresión de resultados. Las cúspides yeyunal y colónica se correlacionaron con los niveles de ingestión de alimentos. No se pudo establecer correlaciones entre la ingestion de medicamentos y excreción de $\mathrm{H}_{2}$.

En lo referente a la relación tallajedad (T/E). los niños de NSE-A tienen distribución percentil $(65,6 \pm 26,3$ ante $28,4 \pm 25,1)$, puntuación $Z$ $(0,58 \pm 0,75$ ante $0,99 \pm 0,92)$ y adecuación al percentil 50 del estándar NCHS (102,6 $\pm 4,5$ ante $96.5 \pm 4.2$ ) significativamente más altos
Tabla 3

Excreción $>20 \mathrm{ppm}$ de hidrógeno espirado entre los 90 y $240 \mathrm{~min}$ (cúspide) en escolares de nivel socioeconómico alto y bajo

\begin{tabular}{|c|c|c|}
\hline & NSE atto $(n: 155)$ & NSE bajo ( $n: 156)$ \\
\hline Presente & $122(78,7 \%)$ & $63(40,4 \%)$ \\
\hline Ausente & $33(21,3 \%)^{* * *}$ & $93(59,6 \%)^{* * *}$ \\
\hline
\end{tabular}

Tabla 4

Patrones de curvas de excreción de hidrógeno espirado en escolares de niveles socioeconómicos alto y bajo

\begin{tabular}{|c|c|c|}
\hline & $\begin{array}{l}\text { NSE allo } \\
(n: 155)\end{array}$ & $\begin{array}{c}\text { NSE bajo } \\
\{n: 156\}\end{array}$ \\
\hline Planas & $29(18.7 \%)^{*}$ & $76(48,7 \%)^{*}$ \\
\hline $\begin{array}{l}<10 \text { ppm a liempo } 0 \\
<20 \text { ppm durante el } \\
\text { estudio ( } 240 \text { min) }\end{array}$ & & \\
\hline
\end{tabular}

Cúspide

$122(78,7 \%)^{* *} \quad 63(40,3 \%)^{* *}$

$>20$ ppm entre los $90 y$

$240 \mathrm{~min}$

$510 \mathrm{ppm}$ a tiempo $0 \quad 65(53,3 \%)^{* * *} \quad 41(65,1 \%)^{* * *}$ $\geq 10$ ppm a tiempo $0 \quad 57(46,7 \%)^{* * *} \quad 22(34,4 \%)^{* * *}$

*: : $x^{2}=28,96$ p $<0,0000001$

**: $\chi^{2}=45,10$ p $<0,00000$

***: $x^{2}=2,36 \mathrm{~ns}$

NSE: nivel sociocconómico

$(p<0,0001)$. No hubo corrclación entre los parámetros antropométricos y la excreción de $\mathrm{H}_{2}$.

\section{Comentario}

En los escolares que participaron en estc estudio, la excreción de más de $20 \mathrm{ppm}$ de $\mathrm{H}_{2}$ en el aire espirado durante los primeros 90 minutos puede ser atribuida a la presencia de una flora anormal en duodeno y yeyuno9, 19.22. Este segmento es habitualmente estéril o presenta recuentos bacterianos que no exceden 103 , principalmente bacterias aerobias o microaerófilas 
Tabla 5

Excreción de hidrógeno en el aire espirado ( $>20 \mathrm{ppm}$ ) durante los primeros 90 min o a los 90 a $240 \mathrm{~min}$ después de ingerir lactulosa en escolares de niveles socioeconómicos alto y bajo, según el tipo de dieta consumida el día anterior al examen

\begin{tabular}{|c|c|c|c|c|}
\hline & \multicolumn{2}{|l|}{ Tiempo 0 a $90 \mathrm{mln}$} & \multicolumn{2}{|c|}{ Tíempo 90 a $240 \mathrm{~min}$} \\
\hline & NSE alto & NSE bajo & NSE alto & NSE bajo \\
\hline yuno nocturno & $\begin{array}{l}6 / 10(60,0 \%) \\
x^{2}=4,35 ; p<0,037(1)\end{array}$ & $17 / 74(23,0 \%)$ & $\begin{array}{l}7 / 10(70,0 \%) \\
p<0,120(3)\end{array}$ & $33 n$ \\
\hline Comida noctuma & $\begin{array}{l}67 / 145(46,2 \%) \\
\chi^{2}=17,51 ; p<0,00001(2)\end{array}$ & $\begin{array}{l}14 / 78(17,9 \%) \\
x^{2}=40,02 ; p<0,00000(2)\end{array}$ & $114 / 145(78,6 \%)$ & $28 / 78(35,9 \%)$ \\
\hline Fibra escasa & $\begin{array}{l}59 / 113(52,2 \%) \\
x^{2}=18,69: p<0,00001(2)\end{array}$ & 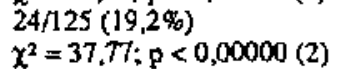 & $88 / 113(77.9 \%)$ & $48 / 125(38,4 \%)$ \\
\hline Fibra moderada & $\begin{array}{l}15 / 40(37,5 \%) \\
x^{2}=1,28 ; p<0,257(2)\end{array}$ & $\hat{6} / 25(24,0 \%)$ & $\begin{array}{l}30 / 40(75,0 \%) \\
\chi^{2}=7,84 ; p<0,005(4)\end{array}$ & $10 / 25(40,0 \%)$ \\
\hline
\end{tabular}

(1) Corrección de Yales

(2) Chi cuadrado

(3) Prueba exacta de Fisher

(4) Mantel-Haenszel

NSE: nivel sociceconómico

provenientes de la cavidad nasal o la orofaringe $^{23}, 24$. En adultos jóvenes de NSE-B se ha encontrado que en la flora detectada en la primera asa del yeyuno predominaban los anaerobios". Esto también fue observado por Klipstein y colaboradores en haitianos asintonáticos 0 con "sprue" tropical, en quienes el intestino alto estaba colonizado por enterobacteriáceas, algunas de las cuales sintetizaban diversos compuestos, entre ellos etanol25. 26. Llama la atención que la proporción de escolares que excretaban $\mathrm{H}_{2}$ en niveles superiores a $20 \mathrm{ppm}$ fue significativamente mayor en el nivel socioeconómico alto. Esto concuerda con la demostración, en preescolares de NSE-A, de tasas mayores de portación asintomática de algunas bacterias que en grupos comparables de nivel bajo'll. Por otra parte, el riesgo de enfernar de fiebre tifoidea parece independiente del nivel socioeconomico y se ha relacionado más bien con las prácticas higiénicas en el hogar ${ }^{27}$. Lo antedicho sugiere que la contaminación microbiológica del ambiente resulta de la interacción de múltiples factores entre los cuales el NSE no es el más importante.

La excreción de más de $10 \mathrm{ppm}$ de $\mathrm{H}_{2}$ en condiciones basales en una proporción de los escolares sugiere la existencia de contaminación intestinal. Nuevamente llama la atención que esto es más frecuente en escolares de NSE-A. Individuos con caries en las piezas dentales o mala higiene oral pueden tener excreciones basales de
$\mathrm{H}_{2}$ elevadas 28.29 . Sin embargo, en nuestro estudio la mayor proporción de piezas con caries, incluso con destrucción de la corona y de enfermedad periodontal, se observó en los nifios de NSE-B. Algunos niños de NSE-A usaban dispositivos para corregir problemas de ortodoncia, que pueden favorecer el desartollo de una flora bucal anormal, pero su higiene oral parecia satisfactoria.

Tal como cabía esperar, una proporción alta de los sujetos estudiados presentaba cúspide colónica, con aumentos de $\mathrm{H}_{2}$ en el aire espirado que excedian $20 \mathrm{ppm}$. Sin embargo, muchos niños no presentaron esta alza, aun cuando algunos tenian cúspide yeyunal. Otros nunca presentaron cúspides de excreción de $\mathrm{H}_{2}$, es decir, sus niveles basales fueron inferiores a $10 \mathrm{ppm}$ y no tuvieron incrementos mayores de $20 \mathrm{ppm}$ después de ingerir lactulosa. Estos sujetos, llamados no productores, han sido también identificados en otros estudios 30,31 .

La dosis de lactulosa usada en este estudio produjo elevaciones definidas en los sujetos que excretaban $\mathrm{H}_{2}$, lo que indica que fue adecuada para los fines de esta investigación. Dosis mayores tienen el inconveniente de producir soluciones hipentónicas o estimular el peristaltismo intestinal, efecto buscado en la investigación de algunas afecciones ${ }^{34}$. Ninguno de los escolares en este estudio presentó molestias abdominales o diarrea. 
La relación talla/edad era más elevada en los sujetos de NSE-A. Se prefirió este parámetro porq̨ue en escolares es más sensible que la relación peso/edad. En los escolares de NSE-A estos parámetros eran comparables a los de países desarrollados ${ }^{35}$. Esto concuerda con otras publicaciones que muestran que el deficit de talla que se observa en sujetos de NSE-B se establece antes de los tres años de edad y genera una diferencia negativa con el NSE-A que no se recupera con el correr de los años. Se considera que dicho déficit en el restiltado acumulativo de aportes dietarios limites y de infecciones repetidas, inciuycndo la colonización anormal del intestino alto ${ }^{1,36}$. Teniendo en cuenta estos antecedentes, en este estudio el cálculo de la dosis de lactulosa se hizo en proporción al peso corporal, por lo que todos los sujetos recibieron dosis comparables.

Como la excreción de $\mathrm{H}_{2}$ en el aire espirado puede ser explicada por procesos fermentativos colónicos provenientes de alimentos ingeridos en días anteriores, se efectuó un recordatorio de 24 horas en todos los sujetos con el fin de calcular en forma aproximada la ingestión de proteínas. energía y fibra dietaria. Se pudo deterninar que la ingestión de fibra era en gencral baja $y$ no se correlacionaba con las cúspides de $\mathrm{H}_{2}$ ni con las etapas en que éstos se producían. Es posible que sustratos endógenos, principalmente el mucus intestinal, también contribuyan a la producción de $\mathrm{H}_{2}$ durante su fermentación ${ }^{32}$. Esto explicaría los resultados obtenidos en un estudio en recién nacidos efcctuado en Australia, en que se observó que la excreción de $\mathrm{H}_{2}$ persistía en niveles altos, incluso duranle el ayuno ${ }^{33}$. Como todas las recolecciones iniciales de aire espirado en nuestro estudio se cfectuaron después de ayunar y a la misma hora del día, es poco probable que los resultados hayan sido modificados por fenómenos de tipo circadiano. Tampoco se pudo correlacionar la excreción de $\mathrm{H}_{2}$ con la ingesta cn los dias previos de medicamentos que pudieran alterar la flora o el peristaltismo intestinales.

La mayor proporción de productores de $\mathrm{H}_{2}$ entre los escolares de NSE-A parece contradecir la hipótesis de que la contaminación microbiológica del ambiente, y por lo tanto del intestino alto, son más frecuentes entre sujetos que viven en peores condiciones de saneamiento. Sín embargo, existe cvidencia que sugicre que sujetos que viven en ambientes contaminados pueden tener una flora bacteriana anormal en el intestino alto que no produce $\mathrm{H}_{2}{ }^{31}$. Es posible que en los sujetos de NSE-A la cantidad o la calidad de su dieta modulen la flora intestinal que coloniza el intestino alto, de manera que una mayor proporción de ella produzca $\mathrm{H}_{2}$ mientras que en el NSE-B se seleccione una llora no productora ${ }^{24,37,38}$.

En resumen, los resultados de este estudio demuestran que en una proporción de los escolares el intestino alto está colonizado, lo que sugiere que la enteropatía ambiental ya está presente en esta edad. El hallazgo de niveles menores de excreción de $\mathrm{H}_{2}$ en los niños de NSE-B nos lleva a proponer la hipótesis que diferencias en las dictas modularian la calidad de la flora que coloniza el segmento duodenoyeyunal.

\section{Resumen}

La contaminación microbiológica del ambiente produce enteropatía ambiental crónica, caracterizada por cambios morfofuncionales en el intestino delgado. En 311 escolares, 155 de NSE-A y 156 NSE-B, se evaluó la colonización bacteriana midiendo $\mathrm{H}_{2}$ en el aire espirado, en muestras en ayunas y cada 15 minutos, durante $240 \mathrm{~min}$, después de ingerir $150 \mathrm{mg} \cdot \mathrm{kg}$ de lactulosa. Una encuesta de salud incluyó información acerca de ingesta de medicamentos y alimentos, consumo de alcohol o tabaco e intolerancia a alimentos. En 30\% de tos niños el nivel basal de excreción de $\mathrm{H}_{2}$ excedía $10 \mathrm{ppm}$. En $18,1 \%$ del NSE-A y $4,5 \%$ del NSE-B $(p<0,0001)$ los máximos de excreción de $\mathrm{H}_{2}$ superaron $20 \mathrm{ppm}$ en los primeros $90 \mathrm{~min}$. Sc detect $\delta$ cúspide colónica $(>20$ ppm) en $78,7 \%$ del NSE-A y $40,4 \%$ del NSE-B (p< $<0,0000$ ). En $18,7 \%$ y $48,7 \%$ del NSE-A y NSE-B, respectivamente, las curvas fueron planas (nivel basal < $10 \mathrm{ppm}$ y aumentos posteriores $<20 \mathrm{ppm}$ ). La enteropatía ambiental crónica ya está presente en escolares. La menor excreción de $\mathrm{H}_{2}$ en el nivel socioeconómico bajo podría explicarse por efectos relacionados con la dieta.

(Palabras clave: enteropatía, contaminación microbiana, excreción respiratoria de $\mathrm{H}_{2}$, lactulosa, escolares, nivel socioeconómico.)

\section{Agradecimientos}

Los autores dcsean expresar sus agradecimientos a los niños que participaron con paciencia y entusiasmo en este 
estudio, a las auloridades y profesiunales de enfermerta de los colegios involucrados. También se desea expresas reco. nocimiento a la Dra. Sylvia Cnuchet a la Enfermera Sra. Mireya Figueron, a la Tecnóloga Médico Sra. Ana María Alieri, a la Sra. Mirta Diaz por el análisis esiadistico y a la Sra. Adriana Diaz Morales.

\section{Referencias}

1. Masa LJ: The children of Santa Marí Cauque. Cambridge: MIT Press, 1978.

2. Troncale FJ, Keusch GT, Miller LH, Otson RA. Buchanan RD: Normal absorption in Thaj subjects with nonspecific jejunal abnormalities. Br Med J 1967: 2: 576.81 .

3. Lindenbown $J_{s}$ Jamiul Alam $A K M, K e n t T H$ : Subclinical small intestinal disease in East Pakistan. Br Med J 1966; 2: 1616-20.

4. Brunser O. Eideiman S, Klipstein FA. Intestinal mucosa in nural Haitians. A comparison between ovest tropical sprue and asymptomatic subjects. Gastroctiterology 1970; $58: 655.68$.

5. Mothan $\mathbf{M}$, Mothan VI, Baker SJ. An electromicroscopic study of jejumal mucosal morphology in control subjects and patients with tropical spne in Southem lndia. Gastroentefology 1975; 68: 17-32.

6. Perea VD, Crut VM, FernJn-Zegarra L, Ballon $R$, Picoago $h$ : Non-specific ileo-jejunitis in Arequipa, Pen. Lancet 1978; 2 : 550-2

7. Thomas $G$, Clain DJ, Wicks ACB: Tropical enterupachy in Rhodesia. Gul 1976; 17: 888-94.

8. Brenser O, Espinoza J, Araya $M$ : Role of the gastrointestinal tract in energy and protein metabolism. Fn: Scrimshaw NS y Schürch $B$, eds. Protein-energy interactions. Lausana: IDECG 1992; 297-305.

9. Briasser O. Arayo M. Espinozo J. Figueroa G. Pacheco 1. Lois f: Chronic environmental enteropathy in a temperate climate. Human Nutr: Clin Nutr 1987; 4IC: 251.61.

10. Espinoza J. Brunter O, Araya M, Egaña $/$, Pachecol, $K$ rause $S$ : The effect of dietary fibre on digestibility of nutrients in a typical diet. En: Rand WM, Uauy $R$ y Scrimshaw NS, eds. Protein-energy-requirement studies in developing countries; resuhs of intemational research. Tokyo: UNU Press, 1984; 294-305.

11. Aruya M, Figueroa $G$, Espinozo J, Zarur X, Brunser $O$ : Acule diarrboea snd the carrier state in Chilean preschoolers of the low and high socio-economic strata. Actn Paedialr Scand 1986: 75: 645-51.

12. Espinoza J. Araya M, Pacheco I, Figueroa G, Spencer $E$. Brunser $O$ : Diarrea aguda en una población infantil marginal de Santiago de Chile. En: AE Lattes, M Farren, J MacDonald, eds. Salud, enfermedad y muerte de los niños en América Latina. Buenos Aires: La Técnica Impresora. 1989; 207-23.

13. Spencer E, Arayo M, Sandino AM, Pacheco I, Brunser $O$ : Faecal excretion of rotavirus and other enteropathegens in newboms of the high and low socio-economic stratum in Santisgo. Chile. Epidemiol Infec 1988; 101: 425-36.

14. Levis MD, Donoldson RM: Use of respiracory hydrogen
$\left(\mathrm{H}_{2}\right)$ excretion to detect carbohydratc malbsoption. J Lab Clin Med 1970; 75: 937.45 .

15. Rhodes JM. Middieton P, Jewell DP: The lactulose bydrogen breath test as a diagnostic test for smallbowel bacterial overgrowth. Scand J Gastroenterol 1979; 14:333-6.

16. Solomons NW: Evaluation of carbohydrate absomption: the hydrogen breath test in clinical practice. Clin Nuar J 1984; $3 ; 71-8$.

17. Pereira SP, Khin-Maung-U, Bolin TD, Duncombe VM, Nyant-Nyant-Wai, Myo-Khit, Linklater JM: A pattem of breath hydrogen excreion suggesting small bowel bacterial overgrowth in Burmese village children. J Pediatr Gastroenterol Nutr 1991: 13:32-8.

18. Graffar $M$ : Etude d'agglomeration en cing cent familles d'une commune de l'agglomeration bruxelloise. Laboratoire de Medecine Sociale, Universitá Libre de Bruxelles. Brussels, Bélgium, 1957.

19. Alvarez $M L$, Wurgafi $F$. Salazar ME: Mediciones do nivel socio-econónico bajo urbano en familias con lactantes Jesnutridos. Arch Latinoam Nutr 1982; 32 : 325-31.

20. Homill PV, Drizd TA, Johnson Cll, et al.: Physiczl growth. National Health Center for Health Statistics percentiles. Am J Clin Nuır 1979; 32:607-29.

21. Kleinbaum DG. Kupper LL, Morgenstern $H$. Epidemiologic research. Príncioles and quantitative methods. New York: Van Nosiraud-Reinhold CO. 1982.

22. Ostrander Cr. Cohen $R$, Jopper AO, Shahin SM. Kerner Jr $J A$, Johnson $J D$. Stevenson $D K$ : Breath hydrogen analysis: a review of the methodologies and clinical applications. I Pedialr Gastroenterol Nutr 1983; $2: 525$. 33.

23. Challacombe $D N$, Richardson $J M$, Anderson CM: Bacterial flora of the upper gastrointestinal tract in infants without diarchoea. Arch Dis Child 1974; 49 264-9.

24. Simon GL, Gurboch SL: Intestinal flora and gastrointestinal function. En: Johnson LR, ed. Physiology of the gastrointestinal tract. Nueva York: Raven Press, 1987; 1729-47.

25. Klipstein FA, Holdeman LV, Corcino JJ, Moore WEC: Enterotoxigenic intestinal bacteria in tropical sprue. Ann Intern Med 1973; 79:632-41.

26. Klipstein FA. Engert RF, Short H: Enterotoxigenicity of colonizing coliform bacteria in tropical sprue and biindloop syndrone. Lancet 1978: 2: 342.4.

27. Alvarez ML, Wurgafi F, Arayo M, Espinoza J: Typhoid fever in children of low and high socioeconomic strala: comparison of hygiene hahits. Rev Saúde Públ., S. Paulo 1990; 24: 108-12.

28. Mostropaolo G, Rees DW: Evaluation of the hysrogen breath test in man: detunition and elimination of the early hydrogen peak. Gut 1987; 28: 72]+5.

29. Thompson $D G, O^{\prime} B$ rien $J D$. Hardie $M M$ : Influence of the oropharyngeal microflora on the measurement of exhaled breath hydrogen. Gasiroentefology 1986; 91 : 853-60.

30. Barr RG, Vanley $I$, Kingsnorth Patterson D, Wooldridge $J$ : Brealh hydrogen excretion in nornal newbom inlants in response to usual feeding partems: evidence for functional lactase insuficiency beyond the first month of life. J Pediatr 1984: 104: 527-33. 
31. Khin-Mawng-U, Tin-Aye, Ku-Tin-Myin, Tin-Oo, Myo. Khin. Thackway SA, Connor SJ, Bolin TD. Duacombe $V M$ : In vitro hydrogen production by enteric bacteria cultured from children with $\mathrm{small}$ bowel baclerial overgrowth. J Pediatr Gastroenterol Nutr 1992; 14: $192-7$.

32. Hoskins LC, Agustines M, MCKee Wa, Boulding ET Kriaesis $M$, Niedermayer $G$ : Mucin degradarion in humsn colon ecosystems. I Clin Invest 1985: 75: 944-53.

33. Miller $J B, B o k d a n M, M C V e a g h P$. Miller $J J$ : Variability of breath hydrogen excretion in breast-fed infants dur. ing the first three months of life. I Pedjatr 1992; 121 . 410-3.

34. Treem WR: Hepatic failure. En: Walker WA, Dutie PR, Hamilion JR, Walker-Smith JA, Watkins JB. Vol. I.
Pefialtic gasuointestinal diseasc. Filadelfia: BC Decket Inc. 1991; 146.92.

35. Folker F: Growth Monitoring: fetus to the first two postnatal years. En: Brunser $O$, Carrazza FR, Graccy $M$, Nichols $B L_{\text {. }}$, Senterre $J$, eds. Clinical nutrition of the young child. Nucva York: Raven Press. 1991: 23-38

36. Martorell $R$, Rivera $J$, Keplowitz $H$ : Consequences of suming in early childhood for adult body size in rural Gualemaja. Ann. Nesılé 1990; 48: 85.92.

37. Gorbach SL, Nahas L, Lerner PI, Weinstein L: Sudies on intestinal microflora. L. Eifects of diet, age, and periodic sampling on numbers of fecal microorganisms in man. Gastroenterology 1967; $53: 845-55$.

38. Finegotd SM, Sutter VL: Fecal flora in differcnt populations, with special reference to diec. $A \mathrm{~m} J$ Clin Nutr 1978; 31 : S1 16-S122. 